\title{
Metodologías, técnicas y herramientas en ingeniería de requisitos: un mapeo sistemático
}

\author{
Methodologies, techniques and tools in requirements \\ engineering: a systematic mapping \\ Dante Carrizo $^{1 *} \quad$ Jorge Rojas ${ }^{1}$ \\ Recibido 31 de marzo de 2016, aceptado 25 de septiembre de 2017 \\ Received: March 31, $2016 \quad$ Accepted: September 25, 2017
}

\begin{abstract}
RESUMEN
Obtener y definir requisitos en etapas tempranas de un proyecto de software es una tarea compleja, por lo que diversos trabajos se han propuesto ayudar en las distintas actividades de la ingeniería de requisitos. Sin embargo, saber en qué áreas de la ingeniería de requisitos existen mayor cantidad de estudios y qué tipo de ellos se han identificado, es crucial para el desarrollo de nuevas investigaciones y búsqueda de soluciones. Este trabajo pretende identificar cuáles son los focos de interés en la literatura científica en ingeniería de requisitos considerando metodologías, técnicas y herramientas, además obtener un registro de estudios orientados a cada actividad de la ingeniería de requisitos. Para el desarrollo de este estudio, se realizó un mapeo sistemático en las bases de datos WOS, IEEEXPLORE y ACM DL. Los resultados obtenidos en 80 artículos seleccionados, se muestra un principal foco de interés en la contribución de técnicas y herramientas tanto en las actividades de educción como análisis de requisitos. La mayoría de los estudios identificados son recientes por lo que aún se requiere validación empírica para ser adoptados por la industria del desarrollo de software.
\end{abstract}

Palabras clave: Ingeniería de requisitos, educción de requisitos, análisis de requisitos, especificación de requisitos, estudio de mapeo sistemático.

\begin{abstract}
Get and set requirements in the early stages of a software project is a complex task, so that several studies have been proposed to assist in the various activities of requirements engineering. However, in what areas of engineering requirements there are more studies and what type of studies have been identified, it is crucial for the development of new research and search for solutions This work tries to identify which are the focus of interest in the scientific literature in requirements engineering considering methodologies, techniques, tools, besides obtaining a record of studies oriented to each activity of the requirements engineering. For the development of this study, a systematic mapping was performed on the WOS, IEEEXPLORE and ACM DL databases. The results obtained in 80 selected articles show a main focus in the contribution of techniques and tools in both elicitation activities and requirements analysis. Most recent studies are identified so empirical validation is still required to be adopted by the software development industry.
\end{abstract}

Keywords: Requirements engineering, requirements elicitation, requirements analysis, requirements specification, systematic mapping study.

1 Departamento de Ingeniería Informática y Ciencias de la Computación. Universidad de Atacama. Av. Copayapu 485. Copiapó, Chile.E-mail: dante.carrizo@uda.cl; jorge.rojas@alumnos.uda.cl

* Autor de correspondencia. 


\section{INTRODUCCIÓN}

La ingeniería de requisitos (en adelante IR) comprende la práctica de educción, análisis, especificación y validación de los requisitos en el desarrollo de software [1]. Estas actividades suelen estar entrelazadas en un proceso iterativo donde ingenieros de software y stakeholders trabajan en conjunto.

El cambio en las preferencias de los stakeholders, restricciones contractuales y el alto desarrollo tecnológico generan un levantamiento y especificación inapropiada de requisitos, lo que puede ser la causa de que muchos proyectos de software fracasen [2]. Por esta razón, los investigadores del software desarrollan nuevas metodologías, técnicas y herramientas para hacer frente a estos problemas.

Para identificar cuáles son temas que están siendo tratados y obtener un registro de propuestas en las distintas actividades de la IR, es necesario tener una visión amplia de la IR. Por este motivo se ha optado por desarrollar un mapeo sistemático de la literatura.

\section{Trabajos previos}

En los años 90, el auge de internet provocó un rápido crecimiento de la demanda de sistemas y mayor focalización en la IR. Con el desarrollo de nuevas metodologías, técnicas y herramientas en IR, revisiones sistemáticas de la literatura (RSL) [3], se han realizado con la finalidad de recolectar información de investigaciones en temas específicos de la IR.

A continuación, se presenta una búsqueda de RSL que se realizó para identificar cuál es el estado del arte en IR.

En 1993 se llevó a cabo una revisión en procesos que eran considerados en ese entonces como cruciales para la IR. En esta revisión, se buscó mejorar mediante técnicas la especificación del lenguaje natural y la rápida creación de prototipos que entreguen resultados rápidos. En algunos casos deben pasar años para que una técnica alcance su madurez y se pueda obtener evidencia significativa del valor, utilidad y aplicabilidad. En ocasiones se corre un riesgo realizar RSL para desarrollar un análisis crítico, como ocurrido en [4], en el que fueron reportados 10 artículos.
En el 2007 una RSL realizó para hacer frente a las tareas de requisitos específicos, principalmente en la obtención y análisis de requisitos, identificar temas actuales y delinear líneas futuras de investigación. Cinco recomendaciones para alcanzar madurez de las tecnologías en IR fueron propuestas. Esta investigación realizó una discusión de los artículos encontrados en cada proceso de IR, pero no especifica qué criterios son utilizados para la inclusión o exclusión de artículos [5].

En el 2009, una RSL desarrollada por [6] se buscó profundizar con la generación de especificaciones de requisitos textuales y documentos de requisitos a partir modelos de ingeniería de software. Se concluyó que existe una falta de herramientas que apoyen el enfoque de "leer y escribir". Por lo general, estas herramientas no son aplicables en la práctica, pero sigue siendo un tema de interés por los investigadores.

En el 2010 fueron reportadas dos investigaciones, en [7] una pequeña revisión de artículos y también encuestas fueron realizadas para desarrollar un marco que facilite la comprensión y colaboración en los procesos de IR con los stakeholders, proporcionando algunos temas ideas interesantes que subyacen a los procesos de IR como la transferencia de conocimiento, confianza entre analistas y stakeholders, desarrollo de modelos compartidos. En [8] se resume la evidencia encontrada en IR de seguridad, especificando técnicas de elicitación, modelado de requisitos, estándar de desarrollo y principal contribución.

A continuación se presenta en la Tabla 1 un resumen de las revisiones identificadas como trabajos previos indicando el tipo de actividad de IR al que fueron focalizadas, el tipo de propuestas que buscan y el número de artículos que seleccionaron.

Este artículo está dividido en 5 secciones. La segunda sección describe la metodología utilizada para el mapeo sistemático. En la tercera sección se realiza una discusión de los resultados. En la cuarta sección se presenta las limitaciones de esta investigación. Finalmente en la quinta sección, se presentan las conclusiones y trabajos futuros.

\section{METODOLOGÍA}

El estudio pretende identificar mayor evidencia sobre metodologías, técnicas y herramientas existentes 
Tabla 1. Resumen de revisiones sistémicas de la literatura.

\begin{tabular}{|c|l|c|l|l|c|}
\hline Ref. & \multicolumn{1}{|c|}{ Título } & Año & \multicolumn{1}{|c|}{$\begin{array}{c}\text { Actividad } \\
\text { en IR }\end{array}$} & \multicolumn{1}{|c|}{$\begin{array}{c}\text { Tipo de } \\
\text { propuestas }\end{array}$} & $\begin{array}{c}\mathbf{N}^{\mathbf{0}} \text { de } \\
\text { artículos }\end{array}$ \\
\hline$[4]$ & "Status report: Requirements engineering" & 1993 & Especificación & Técnicas & 10 \\
\hline$[5]$ & "Research Directions in Requirements Engineering" & 2007 & $\begin{array}{l}\text { Educción, } \\
\text { análisis, } \\
\text { validación, } \\
\text { administración }\end{array}$ & $\begin{array}{l}\text { Metodologías, } \\
\text { técnicas, } \\
\text { herramientas }\end{array}$ & 115 \\
\hline$[6]$ & $\begin{array}{l}\text { "On the generation of requirements specifications } \\
\text { from software engineering models: A systematic } \\
\text { literature review" }\end{array}$ & 2009 & Especificación & Herramientas & 30 \\
\hline$[7]$ & $\begin{array}{l}\text { "An exploration into the process of requirements } \\
\text { elicitation: A grounded approach" }\end{array}$ & 2010 & Educción & $\begin{array}{l}\text { Metodologías, } \\
\text { técnicas }\end{array}$ & 14 \\
\hline$[8]$ & $\begin{array}{l}\text { "A systematic review of security requirements } \\
\text { engineering" }\end{array}$ & 2010 & $\begin{array}{l}\text { Análisis, } \\
\text { especificación, } \\
\text { validación }\end{array}$ & $\begin{array}{l}\text { Metodologías, } \\
\text { técnicas, } \\
\text { herramientas }\end{array}$ & 22 \\
\hline
\end{tabular}

en el proceso de IR del software. Es por esto que se ha optado por un estudio de mapeo sistemático (Systematic Mapping Study, SMS) [9] que permite obtener una visión amplia del campo científico, focos de atención y tendencias de los investigadores. A diferencia de una RSL que es una revisión exhaustiva de evidencia para generar nuevo conocimiento en un área específica.

Los procesos de este mapeo sistemático se pueden observar en la Figura 1.

\section{Definiciones del protocolo}

A continuación se presenta las primeras definiciones para realizar el proceso del mapeo sistemático.

\section{Preguntas de investigación}

Este estudio intenta responder a las siguientes preguntas de investigación. ¿Qué focos de interés existe en investigaciones sobre metodologías, técnicas y herramientas en IR? Además se desea obtener un registro de investigaciones que presentan soluciones a las actividades de educción, análisis, verificación y validación de requisitos.

\section{Alcance de revisión}

Actualmente existe un extenso listado de bases de datos de publicaciones relacionadas con informática. En este estudio nos hemos focalizado en la principal fuente de investigaciones en el área de la ingeniería de software.

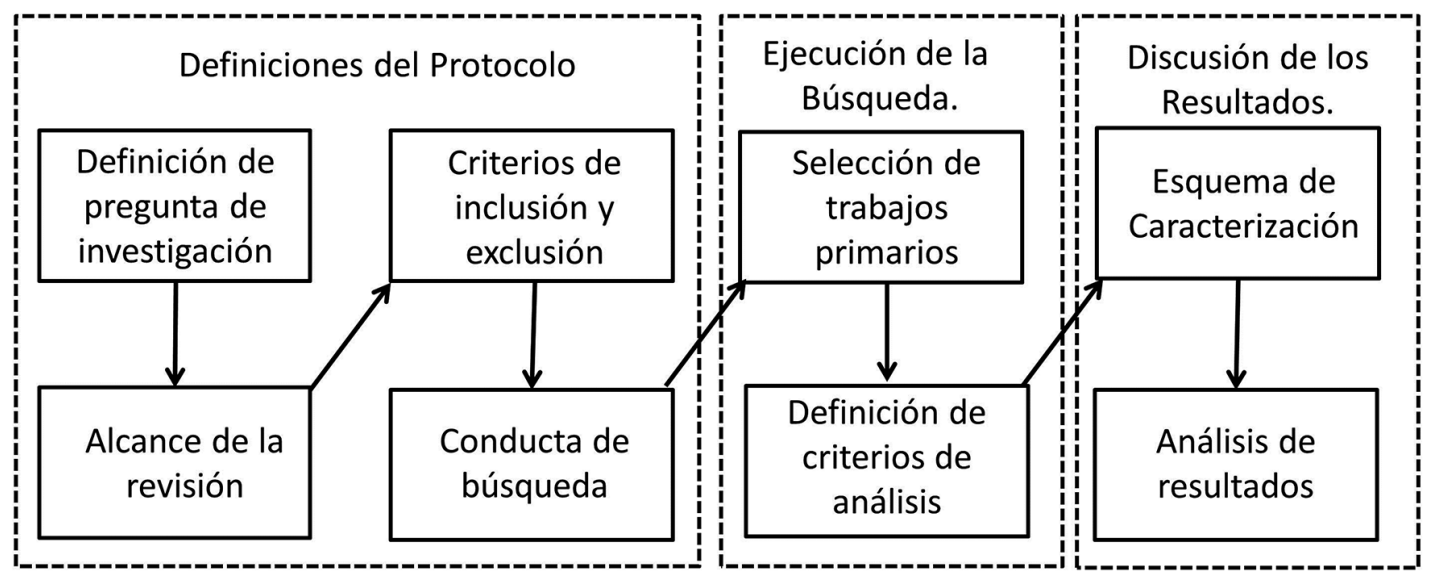

Figura 1. Procesos del mapeo sistemático. 
Para el desarrollo de esta investigación se consideraron las bases de datos IEEXPLORE, ACM DL y WEB OF SCIENCE (WOS). También se realizaron búsquedas oportunistas, referencias de artículos relacionados y otros artículos ya identificados. El período de búsqueda incluye publicaciones desde del auge del internet en 1990 hasta diciembre del 2015.

La cadena de búsqueda utilizada en la base de datos es: (methodology OR techniques OR tools) AND requirements AND engineering.

\section{Criterios de inclusión}

Para la selección de estudios primarios se consideraron los siguientes criterios de inclusión:

- Toda publicación científica es elegible para su inclusión en el estudio si tiene relación con requisitos de software y en donde se propusieran, discutieran o evaluaran metodologías, técnicas y herramientas.

- Se incluyeron estudios cualitativos y cuantitativos realizados por profesionales del software.

- Solo fueron incluidos estudios en inglés, portugués y español.

\section{Criterios de exclusión}

A continuación se presentan los siguientes criterios de exclusión:

- Se excluyeron todos aquellos estudios en los que su enfoque principal se alejara de la IR.

- Los artículos sin diseño de investigación, basados en opiniones de expertos (position paper) que no presentan evidencias y sin una pregunta de investigación definida también fueron excluidos.

\section{Estrategia de búsqueda}

Para identificar los estudios primarios se realizaron los siguientes filtros de revisión:

- $\quad 1^{\text {er }}$ filtro: Revisión de Título.

- $\quad 2^{\text {do }}$ filtro: Revisión de Resumen o Abstract.

- $\quad 3^{\text {er }}$ filtro: Las publicaciones que pasaron el filtro anterior fueron sometidas a una lectura y análisis completo de su contenido.

\section{Ejecución de la búsqueda}

En la siguiente etapa se realiza la selección de los estudios primarios y la definición de los criterios de análisis.

\section{Selección de estudios primarios}

Los resultados de la búsqueda pueden ser observados en la Figura 2, según el formato utilizado por los autores en [10]. Después de aplicar el tercer filtro fueron seleccionadas 78 publicaciones primarias.

\section{Definición de criterios de análisis}

Para el análisis de cada artículo primario, se ha definido un conjunto de criterios que sirven como esquema de clasificación para obtener una vista focalizada de la IR.

Año: Entendiendo como el año de publicación de un artículo de investigación. Buscando identificar si algún proceso en la IR tiene un especial interés a través del tiempo.

Tipo de propuesta: Cualquier artículo encontrado se clasifica de acuerdo si propone o discute algún tipo de metodología, técnica o herramienta/framework en la IR.

Actividad: En la IR se incluyen cuatro actividades de alto nivel según SWEBOK V3 [1].

- Educción: En esta actividad se interactúa con los stakeholders para descubrir sus requisitos, también los requisitos del dominio y documentación existente.

- Análisis: En esta actividad se toma la recolección de requisitos, se clasifican los requisitos relacionados, se definen los servicios que debe proporcionar el sistema, desempeño, requerido, restricciones de hardware, etc.

- Especificación: Los requisitos se documentan y se comprueba la consistencia, integridad, verificabilidad, comprensibilidad, rastreabilidad y adaptabilidad de los requisitos.

- Validación: Debido a que en la actividad anterior intervienen diversos participantes, los requisitos entran en conflicto. Esta actividad se preocupa de validar y verificar que el ingeniero de software ha entendido los requisitos.

\section{Resultados}

A continuación se finaliza la última etapa del mapeo sistemático, donde se presentan los estudios primarios seleccionados y se realiza una discusión de los resultados.

\section{Análisis de estudios primarios}

Por razones de espacio, los estudios primarios se presentan como evidencia del registro con 


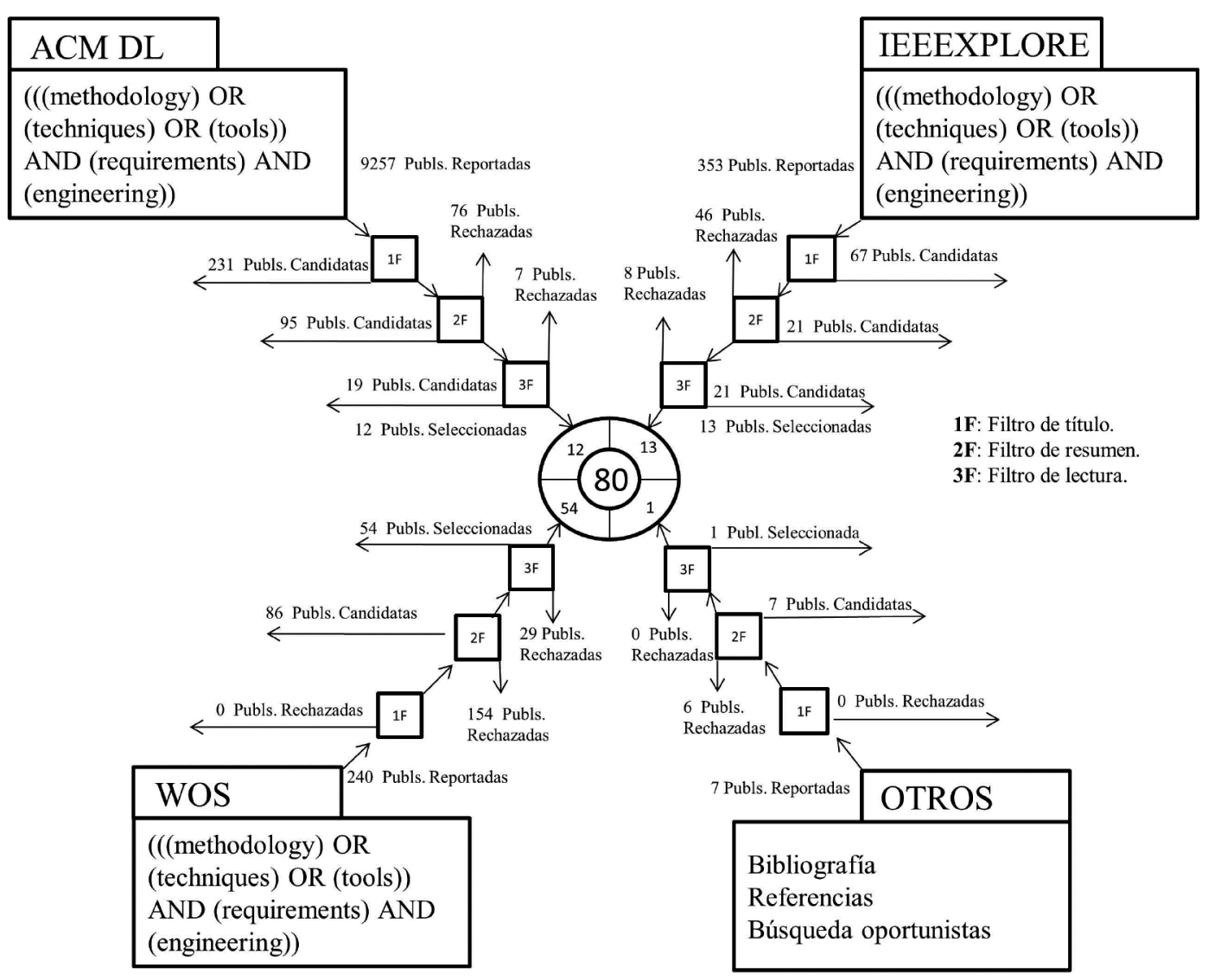

Figura 2. Gráfico de búsqueda de estudios primarios.

información sobre el tipo de propuesta que se realizó, a qué actividad de la IR fue principalmente enfocado y su antigüedad de acuerdo con su fecha de publicación, pueden ser revisados en el link http:// www.diicc.uda.cl/magister/pdf/dc/Anexo_1.pdf

En la Figura 3, como respuesta a la pregunta de investigación general, se observa una visión más completa del mapeo sistemático representado por un gráfico burbuja. En él, clasificamos los estudios primarios de acuerdo con los criterios de análisis mencionados anteriormente. Además, se puede apreciar que existe una atención en el desarrollo de técnicas y herramientas para apoyar la actividad de educción y análisis de requisitos del software.

\section{Discusión por criterios}

A continuación se presenta una discusión por los criterios definidos anteriormente para la clasificación y como respuesta a la pregunta de investigación, ¿Qué focos de interés existe en investigaciones sobre metodologías, técnicas y herramientas en IR?

\section{Ingeniería de requisitos en el tiempo}

Cerca de 8 años tuvieron que transcurrir para identificar investigaciones que hagan frente a las diversas problemáticas existentes en la IR. Es posible que no se hayan encontrado investigaciones anterior a 1998 debido a no se utilizaron sinónimos como administración de requisitos o requisitos de software, en la cadena de búsqueda. A principios del 2002 los estudios primarios encontrados son enfocados en su mayoría al proceso de educción y análisis de requisitos. Si bien, este proceso de educción es crucial para el futuro del proyecto, aún sigue siendo un problema fundamental obtener los requisitos correctos de los stakeholders.

\section{Tipos de propuestas}

A continuación en la Figura 4, se observa un gráfico de barras registrando la cantidad de metodologías 


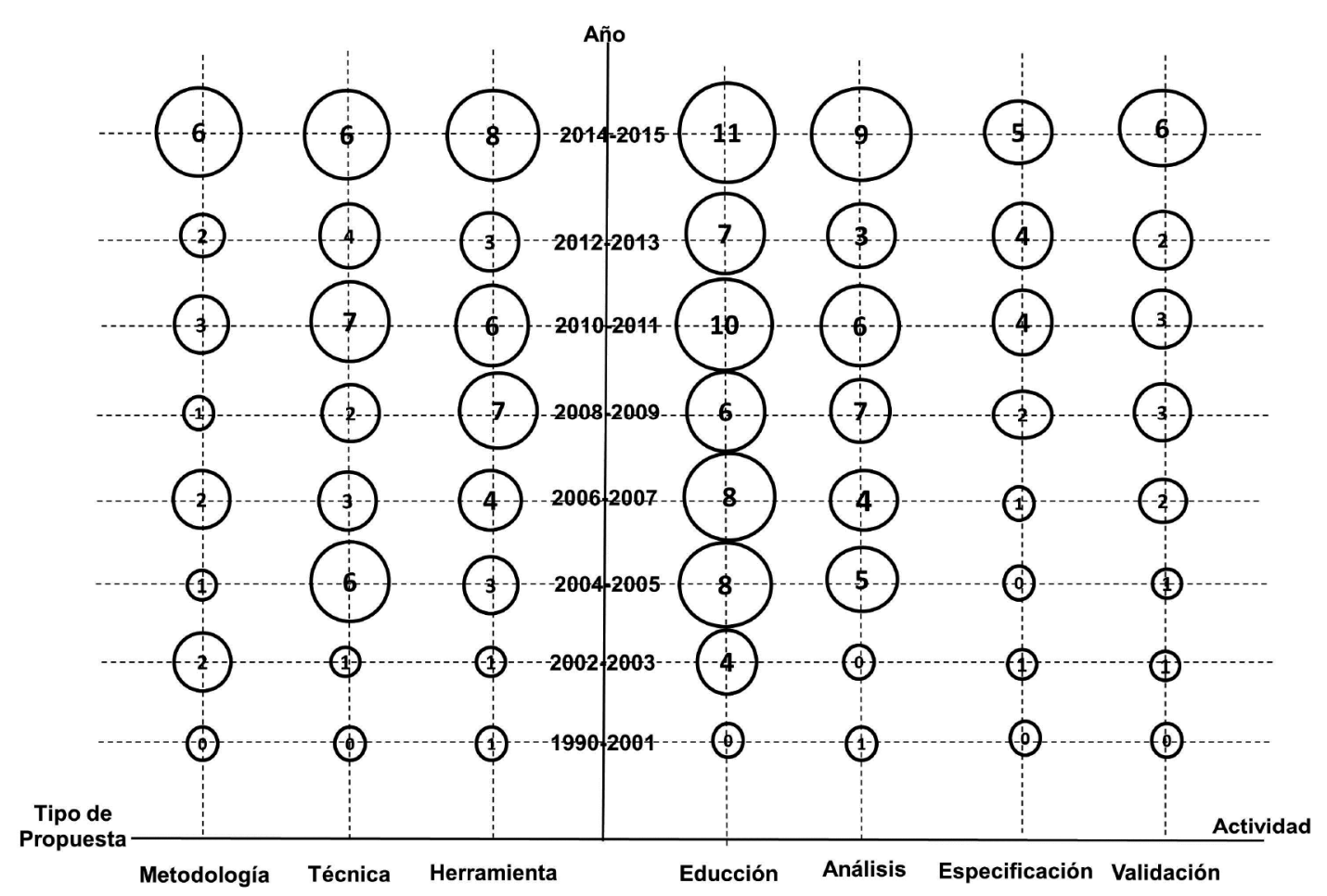

Figura 3. Gráfico de burbuja de los estudios primarios.

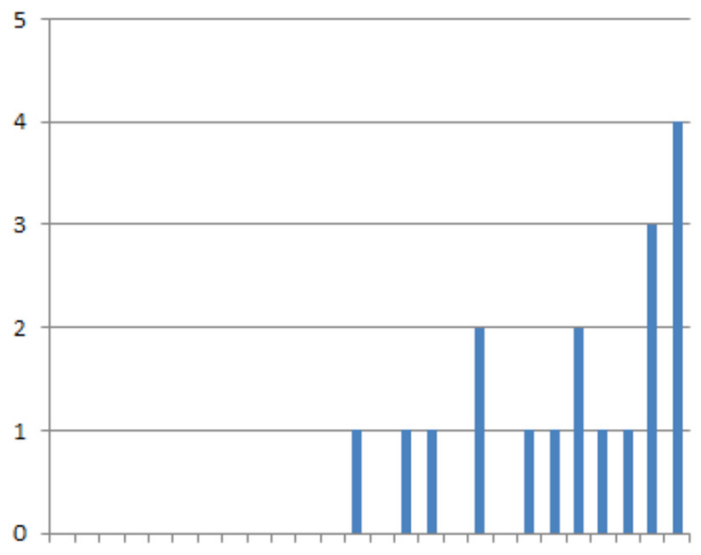

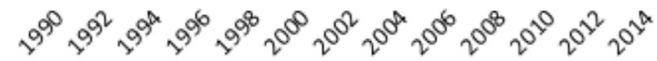

Figura 4. Número de artículos sobre metodologías en IR.

en ingeniería de requisitos propuestas en los 25 años de investigación.

Antes de los 90 variadas metodologías fueron propuestas en el área de la inteligencia artificial para el desarrollo de sistemas expertos [11], pero estas metodologías fueron incorporadas años más tardes en la ingeniería de software. Es por ello que del registro obtenido, las metodologías propuestas o evaluadas en la ingeniería de software son principalmente enfocadas en agentes para el entendimiento organizacional y orientado a metas para ayudar tanto en el modelado como en el análisis con la utilización de árboles.

Con un total de 29 publicaciones reportadas, se puede apreciar que se muestra un interés por los investigadores en desarrollar técnicas para ayudar en el proceso de la IR. En la Figura 5, se puede

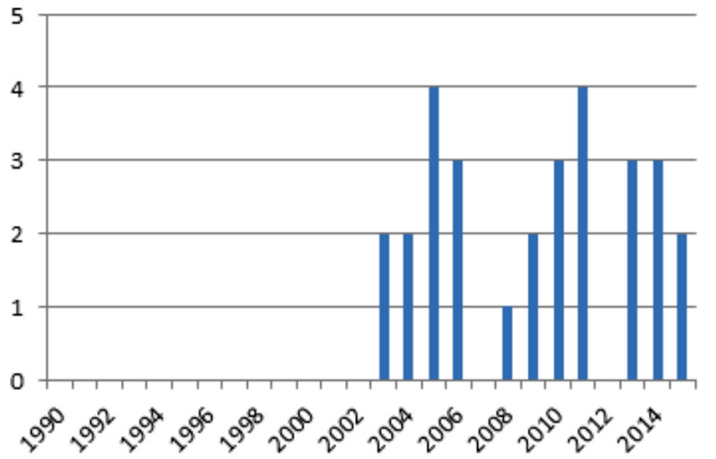

Figura 5. Número de artículos sobre técnicas en IR. 
apreciar la cantidad de técnicas propuestas IR mediante el tiempo.

Teniendo registros de técnicas desde el 2003 en adelante, aún se requiere más validación empírica para ser adoptadas por la industria desarrolladora de software, ya que estas técnicas son utilizadas principalmente en un estudio de caso con un ambiente previamente seleccionado.

En la Figura 6 se presenta la cantidad de herramientas desarrolladas en la IR en 25 años de investigación.

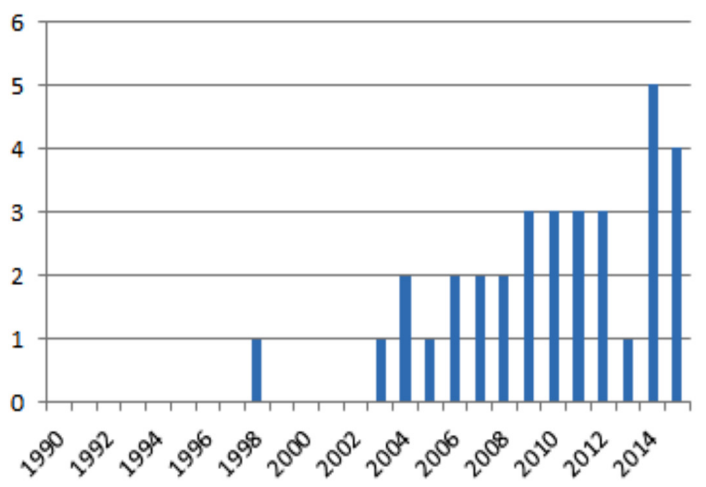

Figura 6. Número de artículos sobre herramientas en IR.

Con un total de 33 publicaciones con propuestas de herramientas, al igual que las publicaciones de técnicas son relativamente nuevas. Estas herramientas son enfocadas en su mayoría a facilitar las actividades de educción y análisis de requisitos.

\section{DISCUSIÓN DE LOS RESULTADOS}

En acuerdo con las RSL, las investigaciones en técnicas y herramientas en educción de requisitos, buscan principalmente facilitar la compresión y obtención con los stakeholders en lenguaje natural $[4-5,7]$. Pero esto es diferente en los resultados obtenidos en nuestro mapeo sistemático. Las técnicas son alineadas a los objetivos del negocio, buscando reutilizar requisitos utilizando benchmarking [12] y automatizando esta tarea, [23, 36, 39, 78, 91].

Poco se pronuncia en las RLS, pero se puede apreciar en este SMS la cantidad de investigaciones que utilizan el paradigma de desarrollo dirigidos por modelos (MDD), específicamente en el tratamiento explícito de metas, más conocido como IR orientada a metas (siglas en inglés, GORE). En GORE se definen metamodelos que contribuyen a aumentar el grado de cobertura y sistematización del proceso de definición de requisitos. Este método es utilizado en [30, 46, 48, 67, 69]. Demostrando un claro interés por los investigadores en esta área.

Un punto a considerar es que tanto en las RSL y el mapeo sistemático es notorio la ausencia de investigaciones en la que se estudie la gestión de requisitos. Esta área de la IR sigue siendo un problema para los ingenieros principalmente en grandes proyectos de software.

\section{LIMITACIONES}

Se puede señalar que una de las limitantes de esta investigación es que no se utilizaron sinónimos en la cadena de búsqueda lo que podría no identificar investigaciones en donde la ingeniería de requisitos es nombrada como administración de requisitos o requisitos del software.

\section{CONCLUSIONES Y TRABAJO FUTURO}

Lograr descubrir, analizar y definir los requisitos en etapas tempranas del software es fundamental en la IR. Diversos estudios se han llevado a cabo para mitigar problemas que surgen en las actividades de educción, análisis, especificación y validación de requisitos. Pero la mayor concentración de propuestas científicas reportadas son en el desarrollo de técnicas y herramientas para las actividades educción y análisis.

Aunque existen numerosas herramientas que facilitan la comprensión de requisitos de los stakeholders y el modelados de estos, aún falta por crear herramientas que ayuden en la trazabilidad de los requisitos.

Es importante destacar que aun teniendo gran cantidad de propuestas en técnicas en IR, es fundamental la correcta selección de estas dependiendo el tipo de proyecto como lo destacan en [23].

Como trabajo futuro se realizará una investigación tipo survey para identificar cuáles son las brechas existentes entre la literatura científica y la industria latinoamericana del software en la IR. 


\section{REFERENCIAS}

[1] P. Bourque and R.E. Fairley. "Guide to the software engineering body of knowledge". IEEE Computer Society Press. Version 3.0. 2014. ISBN: 07695516619780769551661.

[2] B, Mobasher and J. Cleland-Huang. "Recommender Systems in Requirements Engineering". AI Magazine. Vol. 32, pp. 81-89. 2011. DOI: https://doi.org/10.1609/ aimag.v32i3.2366.

[3] S.E. Group. "Guidelines for performing Systematic Literature Reviews in Software Engineering”. EBSE Technical Report. 2007.

[4] P. Hsia, A. Davis and D. Kung. "Status report: Requirements engineering". IEEE Software. Vol. 10, pp. 75-79. 1993. DOI: 10.1109/52.241974.

[5] B.H.C. Cheng and J.M. Atlee. "Research Directions in Requirements Engineering". Future of Software Engineering (FOSE'07), pp. 285-303. 2007. DOI: 10.1109/FOSE. 2007.17.

[6] J. Nicolás and A. Toval. "On the generation of requirements specifications from software engineering models: A systematic literature review". Information and Software Technology. Vol. 51, pp. 1291-1307. 2009. DOI: 10.1016/j.infsof.2009.04.001.

[7] S. Chakraborty, S. Sarker and S. Sarker. "An exploration into the process of requirements elicitation: A grounded approach". Journal of the Association for Information Systems. Vol. 11, pp. 212. 2010. ISSN: 15369323.

[8] D. Mellado, C. Blanco L.E. Sánchez. and E. Fernández. "A systematic review of security requirements engineering". Computer Standards \& Interfaces. Vol. 32, pp. 153-165. 2010. DOI: doi.org/10.1016/j. csi.2010.01.006.

[9] D. Carrizo and J. Rojas. "Clasificación de prácticas de educción de requisitos en desarrollos ágiles: Un mapeo sistemático". Ingeniare. Revista chilena de ingeniería. Vol. 24, p. 10. 2016. DOI: dx.doi.org/10.40.67/ S0718-33052016000400010.

[10] D. Carrizo and C. Ortiz. "Modelos del proceso de educción de requisitos: Un mapeo sistemático". Revista Científica Ingeniería y Desarrollo. Vol. 34, pp. 184-203. 2016. DOI: http://dx.doi.org/10.14482/inde.34.1.7958.
[11] M. Daneva, D. Damian, A. Marchetto and O. Pastor. "Empirical research methodologies and studies in Requirements Engineering: How far did we come?". Journal of systems and software. Vol. 95, pp. 1-9. 2014. DOI: https://doi.org/10.1016/j.jss.2014.06.035.

[12] M.S. Pang, W. Suh, J. Kim and H. Lee. "A Benchmarking-Based Requirement Analysis Methodology for Improving Web Sites". International Journal of Electronic Commerce. Vol. 13, pp. 119-162. 2009. DOI: 10.2753/ JEC1086-4415130305.

[13] H. Yang and J. Tang. "A three-stage model of requirements elicitation for Web-based information systems". Ind. Manag. Data Syst. Vol. 103, pp. 398-409, 2003. ISSN: 0007-6929, ZDB-ID 7633907.

[14] L. Al-Salem and A. Abu Samaha. "Eliciting Web application requirements - an industrial case study". J. Syst. Softw. Vol. 80, pp. 294313, 2007. DOI: 10.1016/j.jss.2006.05.005.

[15] F. Benitti and R. da Silva. "Evaluation of a systematic approach to requirements reuse". Evaluation of a Systematic Approach to Requirements Reuse. Vol. 19, pp. 254-280, 2013. DOI: $10.3217 /$ jucs-019-02-0254.

[16] C. Gralha, J. Araújo and M. Goulão. "Metrics for measuring complexity and completeness for social goal models". Information Systems. Vol. 53, pp. 346-362, 2015. DOI: https://doi. org/10.1016/j.is.2015.03.006.

[17] A. Ullah and R. Lai. "Modeling Business Goal for Business/It Alignment Using Requirements Engineering". Journal of Computer Information Systems. Vol. 51, pp. 21-28, 2011. DOI: 10.1080/08874417.2011.11645482.

[18] M. Sen and S. Jain. "An Agile Technique for Agent Based Goal Refinement to Elicit Soft Goals in Goal Oriented Requirements Engineering". In Advanced Computing and Communications. pp. 41-47, 2007. DOI: 10.1109/ADCOM.2007.34.

[19] E. Casagrande, S. Woldeamlak, W. Woon, H. Zeineldin and D. Svetinovic. "NLP-KAOS for systems goal elicitation: Smart metering system case study". IEEE Transactions on Software Engineering. Vol. 40, pp. 941-956, 2014. DOI: 10.1109/TSE.2014.2339811.

[20] J. Kim, J. Kim, S. Park and V. Sugumaran. "A multi-view approach for requirements analysis using goal and scenario". 
Industrial Management \& Data Systems. Vol. 104, pp. 702-711, 2004. DOI: $10.1108 / 02635570410567694$.

[21] M. Sen and K. Hemachandran. "Elicitation of goals in requirements engineering using agile methods". Computer Software and Applications Conference Workshops (COMPSACW), pp. 263-268, 2010. DOI: 10.1109/COMPSACW.2010.53.

[22] S. Lauesen and J. Vium. "Communication gaps in a tender process". Requirements Engineering. Vol. 10, pp. 247-261, 2005. DOI: https://doi.org/10.1007/s00766-005-0009-2.

[23] D. Carrizo, O. Dieste and N. Juristo. "Systematizing requirements elicitation technique selection". Information and Software Technology. Vol. 56, pp. 644669, 2014. DOI: https://doi.org/10.1016/j. infsof.2014.01.009.

[24] J.A.R.P. Sardinha, R. Choren, V.T. da Silva, R. Milidiú and C.J. de Lucena. "A combined specification language and development framework for agent-based application engineering". Journal of Systems and Software. Vol. 79, pp. 1565-1577, 2006. DOI: https://doi.org/10.1016/j.jss.2006.02.018.

[25] J. Choi, R.I. Arriaga, H. Moon and E. Lee. "A Context-Driven Development Methodology for Context-Aware Systems". Convergence and Hybrid Information Technology. Vol. 6935, pp. 429-436. DOI: https://doi. org/10.1007/978-3-642-24082-9_53.

[26] S. Islam, H. Mouratidis and J. Jürjens. "A framework to support alignment of secure software engineering with legal regulations". Software \& Systems Modeling. Vol. 10, pp. 369-394, 2011.DOI: https://doi.org/10.1007/ s10270-010-0154-z.

[27] N. Gorse, P. Bélanger, A. Chureau, E.M. Aboulhamid and Y. Savaria. "A high-level requirements engineering methodology for electronic system-level design". Computers \& Electrical Engineering. Vol. 33, pp. 249268, 2007. DOI: https://doi.org/10.1016/j. compeleceng.2007.02.004.

[28] J. Kim, S. Park and V. Sugumaran. "Improving use case driven analysis using goal and scenario authoring: A linguistics-based approach". Data \& Knowledge Engineering. Vol. 58, pp. 21-46, 2006. DOI: https://doi. org/10.1016/j.datak.2005.05.006.
[29] A. Perini, A. Susi and P. Avesani. "A machine learning approach to software requirements prioritization". IEEE Transactions on Software Engineering. Vol. 39, pp. 445-461, 2013. DOI: 10.1109/TSE.2012.52.

[30] M. Rossi and T. Tuunanen. "A method and tool for wide audience requirements elicitation and rapid prototyping for mobile systems". Conceptual Modeling for Advanced Application Domains, pp. 629-640, 2004. DOI: https:// doi.org/10.1007/978-3-540-30466-1_58.

[31] M. Deng, K. Wuyts, R. Scandariato, B. Preneel and W. Joosen. "A privacy threat analysis framework: Supporting the elicitation and fulfillment of privacy requirements". Requirements Engineering. Vol. 16, pp. 3-32, 2011. DOI: https://doi.org/10.1007/ s00766-010-0115-7.

[32] R. Fuentes, J.J. Gómez and J. Pavón. "A sociological framework for multi-agent systems validation and verification". Conceptual Modeling for Advanced Application Domains. Vol. 3289, pp. 458-469, 2004. DOI: https:// doi.org/10.1007/978-3-540-30466-1_42.

[33] G. Elahi, E. Yu and N. Zannone. "A vulnerability-centric requirements engineering framework: Analyzing security attacks, countermeasures, and requirements based on vulnerabilities". Requirements Engineering. Vol. 15, pp. 41-62, 2010. DOI: https://doi. org/10.1007/s00766-009-0090-z.

[34] D. Jannach and G. Kreutler. "Advisor suite: A tool for rapid development of maintainable Online sales advisory systems". Web Engineering. Vol. 3140, pp. 266-270, 2004. DOI: https://doi. org/10.1007/978-3-540-27834-4_34.

[35] B. Henson, C. Barnes, R. Livesey, T. Childs and K. Ewart. "Affective Consumer Requirements: A Case Study of Moisturizer Packaging". Concurrent Engineering. Vol. 14, pp. 187-196, 2006. DOI: https:// doi.org/10.1177/1063293X06068358.

[36] A. Perini, A. Pistore, M. Roveri \& M. Susi. "Agent-oriented modeling by interleaving formal and informal specification". International Workshop on Agent-Oriented Software Engineering, pp. 36-52, 2003. DOI: https://doi.org/10.1007/978-3-540-24620-6_3.

[37] L. Mich, C. Anesi and D.M. Berry. "Applying a pragmatics-based creativity-fostering 
technique to requirements elicitation". Requirements Engineering. Vol. 10, pp. 262275, 2005. DOI: https://doi.org/10.1007/ s00766-005-0008-3.

[38] Z. Jin, D. Bell, F. Wilkie and D.G. Leahy. "Automated Requirements Elicitation: Combining a Model-Driven Approach With Concept Reuse". International Journal of Software Engineering and Knowledge Engineering. Vol. 13, pp. 53-82, 2003. DOI: https://doi.org/10.1142/S0218194003001147.

[39] J. Jiao and C. Chen. "Customer Requirement Management in Product Development: A Review of Research Issues". Concurrent Engineering. Vol. 14, pp. 173-185, 2006. DOI: https://doi.org/10.1177/1063293X06068357.

[40] P. Beynon and S. Holmes. "Design breakdowns, scenarios and rapid application development". Information and Software Technology. Vol. 44, pp. 579-592, 2002. DOI: https:// doi.org/10.1016/S0950-5849(02)00078-2.

[41] E. Bagheri and F. Ensan. "Dynamic decision models for staged software product line configuration". Requir. Eng. Vol. 19, pp. 187212, 2014. DOI: https://doi.org/10.1007/ s00766-013-0165-8.

[42] T. Srivatanakul, J.A. Clark and F. Polack. "Effective security requirements analysis: HAZOP and use cases". Information Security. Vol. 3225, pp. 416-427, 2004. DOI: https:// doi.org/10.1007/978-3-540-30144-8_35.

[43] T. Al Balushi, P. Sampaio and P. Loucopoulos. "Eliciting and prioritizing quality requirements supported by ontologies: A case study using the ElicitO framework and tool". Expert System. Vol. 30, pp. 129-151, 2013. DOI: 10.1111/j.1468-0394.2012.00625.x.

[44] M. Montali, P. Torroni, N. Zannone, P. Mello and V. Bryl. "Engineering and verifying agent-oriented requirements augmented by business constraints with B-Tropos". Autonomous Agents and Multi-Agent Systems. Vol. 23, pp. 193-223, 2011. DOI: https://doi.org/10.1007/s10458-010-9135-4.

[45] A. Morkevicius and S. Gudas. "Enterprise Knowledge Based Software Requirements Elicitation". Information Technology and Control. Vol. 40, pp. 181-190, 2011. DOI: http://dx.doi.org/10.5755/j01.itc.40.3.626.

[46] A. Sutcliffe, S. Thew and P. Jarvis. "Experience with user-centred requirements engineering".
Requirements Engineering. Vol. 16, pp. $267-$ 280, 2011. DOI: https://doi.org/10.1007/ s00766-011-0118-z.

[47] J. Mouratidis \& H. Jurjens. "From goal-driven security requirements engineering to secure design". International Journal of Intelligent Systems. Vol. 25, pp. 813-840, 2010. DOI: 10.1002/int.20432.

[48] C. Damas, B. Lambeau, P. Dupont and A. Van Lamsweerde. "Generating annotated behavior models from end-user scenarios". IEEE Transactions on Software Engineering. Vol. 31, pp. 1056-1073, 2005. DOI: 10.1109/ TSE.2005.138.

[49] A. Casamayor, D. Godoy and M. Campo, "Identification of non-functional requirements in textual specifications: A semi-supervised learning approach". Information and Software Technology. Vol. 52, pp. 436-445, 2010. DOI: https://doi.org/10.1016/j.infsof.2009.10.010.

[50] H. Kaiya, D. Shinbara, J. Kawano and M. Saeki. "Improving the detection of requirements discordances among stakeholders". Requirements Engineering. Vol. 10, pp. 289-303, 2005. DOI: https:// doi.org/10.1007/s00766-005-0017-2.

[51] A.K. Thurimella and B. Bruegge. "Issuebased variability management". Information and Software Technology. Vol. 54, pp. 933950, 2012. DOI: https://doi.org/10.1016/j. infsof.2012.02.005.

[52] K. Pitula and T. Radhakrishnan. "On eliciting requirements from end-users in the ICT4D domain". Requirements Engineering. Vol. 16, pp. 323-351, 2011. DOI: https:// doi.org/10.1007/s00766-011-0127-y.

[53] J. Jwo and Y. Cheng. "Pseudo software: A mediating instrument for modeling software requirements". Journal of Systems and Software. Vol. 83, pp. 599-608, 2010. DOI: https://doi.org/10.1016/j.jss.2009.10.042.

[54] S. Liaskosm S.A. McIlraith, S. Sohrabi and J. Mylopoulos. "Representing and reasoning about preferences in requirements engineering". Requirements Engineering. Vol. 16, pp. 227-249, 2011. DOI: https:// doi.org/10.1007/s00766-011-0129-9.

[55] R. Fuentes, J. Gómez and J. Pavón. "Requirements elicitation and analysis of multiagent systems using activity theory". IEEE Transactions on Systems, Man, and 
Cybernetics - Part A: Systems and Humans. Vol. 39, pp. 282-298, 2009. DOI: 10.1109/ TSMCA.2008.2010747.

[56] T. Miller, B. Lu, L. Sterling, G. Beydoun and $\mathrm{K}$. Taveter. "Requirements elicitation and specification using the agent paradigm: The case study of an aircraft turnaround simulator". IEEE Transactions on Software Engineering. Vol. 40, pp. 1007-1024, 2014. DOI: 10.1109/TSE.2014.2339827.

[57] J. Fuentes, R. Gómez \& J. Pavón. "Requirements Elicitation for agent-based applications". International Workshop on Agent-Oriented Software Engineering. Vol. 3950, pp. 40-53, 2005. DOI: https:// doi.org/10.1007/11752660_4.

[58] J. Carrillo De Gea, J. Nicolás, J. Fernández, A. Toval, C. Ebert and A. Vizcaíno. "Requirements engineering tools: Capabilities, survey and assessment". Information and Software Technology. Vol. 54, pp. 1142-1157, 2012. DOI: https://doi.org/10.1016/j.infsof.2012.04.005.

[59] N. Kiyavitskaya and N. Zannone. "Requirements model generation to support requirements elicitation: The Secure Tropos experience". Vol. 15, pp. 149-173, 2008. DOI: https://doi.org/10.1007/s10515-008-0028-6.

[60] D. Mellado, H. Mouratidis and E. Fernández. "Secure Tropos framework for software product lines requirements engineering". Computer Standards \& Interfaces. Vol. 36, pp. 711-722, 2014. DOI: https://doi. org/10.1016/j.csi.2013.12.006.

[61] P. Sawyer, P. Rayson and K. Cosh. "Shallow knowledge as an aid to deep understanding in early phase requirements engineering". IEEE Transactions on Software Engineering. Vol. 31, pp. 969-981, 2005. DOI: 10.1109/ TSE.2005.129.

[62] S. Somé. "Supporting use case based requirements engineering". Information and Software Technology. Vol. 48, pp. 43-58, 2006. DOI: https://doi.org/10.1016/j. infsof.2005.02.006.

[63] S. Uchitel, G. Brunet and M. Chechik. "Synthesis of partial behavior models from properties and scenarios". IEEE Transactions on Software Engineering. Vol. 35, pp. 384406, 2009. DOI: 10.1109/TSE.2008.107.

[64] M. El-Attar. "Towards developing consistent misuse case models". Journal of Systems and
Software. Vol. 85, pp. 323-339, 2012. DOI: https://doi.org/10.1016/j.jss.2011.08.023.

[65] R. Fuentes, J.J. Gómez and J. Pavón. "Understanding the human context in requirements elicitation". Requirements Engineering. Vol. 15, pp. 267-283, 2010. DOI: https://doi.org/10.1007/s00766-009-0087-7.

[66] C. Durugbo and J. Riedel. "Viewpointparticipation-technique: A model of participative requirements elicitation". Concurrent Engineering. Vol. 21, pp. 3-12, 2013. DOI: https://doi.org/10.1177/1063293X12474029.

[67] S. Miyazaki, N. Mead and J.Zhan. "Computeraided privacy requirements elicitation technique". Asia-Pacific Services Computing Conference, pp. 367-372, 2008. DOI: 10.1109/APSCC.2008.263.

[68] J. George and O. Mohamed. "A Methodology To Generate Evenly Distributed Input Stimuli By Clustering Of Variable Domain". Computer Design (ICCD), pp. 605-612, 2015. DOI: 10.1109/ICCD.2015.7357171.

[69] R. Jardim and V. Taseva. "Application of SQuaRE and Generalized Nets for extended validation of CE systems". Technology Management Conference (ICE), pp. 1-8, 2009. DOI: 10.1109/ITMC.2009.7461371.

[70] O. Maksimenkova and A. Neznanov. "Blended Learning in Software Engineering Education: the Application Lifecycle Management Experience with Computer-Supported Collaborative Learning", pp. 655-662, 2015. DOI: 10.1109/ICL.2015.7318104.

[71] F. Regazzoni. "Design Methodologies for Securing Cyber-Physical Systems". Hardware/Software Codesign and System Synthesis (CODES+ISSS), pp. 30-36, 2015. DOI: 10.1109/CODESISSS.2015.7331365.

[72] G. Casale, D. Ardagna, M. Artac, F. Barbier, E. Di Nitto, A. Henry, G. Iuhasz, C. Joubert. J. Merseguer, V. Munteanu, J. Pérez, D. Petcu, M. Rossi, C. Sheridan and I. Spais. "DICE: Quality-Driven Development of DataIntensive Cloud Applications". Modeling in Software Engineering (MiSE), pp. 78-83, 2015. DOI: 10.1109/MiSE.2015.21.

[73] W. Ecker, M. Velten, L. Zafari and A. Goyal, "Metasynthesis for Designing Automotive SoCs". Design Automation Conference (DAC), pp. 1-6, 2014. DOI: 10.1109/ DAC.2014.6881398. 
[74] M. Ravindra, "Novel methodology for requirements to design traceability of onboard". Advances in Electronics, Computers and Communications (ICAECC). pp. 1-6, 2014. DOI: 10.1109/ICAECC.2014.7002386.

[75] N. Bombieri, F. Fummi, V. Guarnieri, G. Pravadelli, F. Stefanni, T. Ghasempouri, M. Lora, G. Auditore and M. Marcigaglia. "On the reuse of RTL assertions in System C TLM verification", pp. 1-6, 2014. DOI: 10.1109/LATW.2014.6841903.

[76] J. Crane, B. Hamilton and L. Brownlow. "Optimization of Multi-Satellite Systems Using Integrated Model Based System Engineering ( MBSE) Techniques". Systems Conference (SysCon). pp. 206-211, 2015. DOI: 10.1109/SYSCON.2015.7116753.

[77] A. Nicolás and P. Sánchez. "Parallel native-simulation for multi-processing embedded systems". Digital System Design (DSD), pp. 543-546, 2015. DOI: 10.1109/ DSD.2015.75.

[78] I. Ifip. "Semi-Automatic Generation of Extended Finite State Machines from Natural Language Standard Documents". Dependable Systems and Networks Workshops (DSNW), pp. 45-50, 2015. DOI: 10.1109/ DSN-W.2015.17.

[79] J. Horkoff, R. Salay, M. Chechik, A. Di Sandro and C. Science. "Supporting Early Decision-Making in the Presence of Uncertainty". Requirements Engineering Conference (RE), pp. 33-42, 2014. DOI: 10.1109/RE.2014.6912245.

[80] J. Rekoske. "A Practical Extension to the VORD Model". ACM SIGSOFT Software Engineering Notes. Vol. 31, pp. 1-5, 2006. DOI: $10.1145 / 1163514.1163532$.

[81] D. Ferreira and A. Rodrigues. "A Requirements Specification Case Study with ProjectITStudio/Requirements". Proceedings of the 2008 ACM symposium on Applied computing, pp. 656-657, 2008. DOI: 10.1145/1363686.1363843.

[82] M. Saeki. "A Tool for Attributed GoalOriented Requirements Analysis". Automated Software Engineering, pp. 674-676, 2009. DOI: 10.1109/ASE.2009.34

[83] A. Kalavade, B. Labs and B. Labs. "A Tool for Performance Estimation of Networked Embedded End-Systems". Proceedings of the 35th annual Design Automation Conference, pp. 257-262, 1998. DOI: 10.1145/277044.277116.

[84] P. Jain, K. Verma, A. Kass, R.G. Vasquez, W. Fernando and S. Jose. "Automated Review of Natural Language Requirements Documents: Generating Useful Warnings with User-extensible Glossaries Driving a Simple State Machine". Proceedings of the 2nd India software engineering conference, pp. 37-45, 2009. DOI: $10.1145 / 1506216.1506224$.

[85] G. Rempel. "Defining Standards for Web Page Performance in Business Applications". Proceedings of the 6th ACM/SPEC International Conference on Performance Engineering, pp. 245-252, 2015. DOI: $10.1145 / 2668930.2688056$.

[86] A. Coronato and G.D.E. Pietro. "Formal Specification of Wireless and Pervasive Healthcare Applications". ACM Transactions on Embedded Computing Systems (TECS). Vol. 10, pp. 12, 2010. DOI: 10.1145/1814539.1814551.

[87] J. García, J. Escalona, M. Ravel and M. Urbieta. "NDT-Merge: A future tool for conciliating Software Requirements in MDE Environments". Proceedings of the 14th International Conference on Information Integration and Web-based Applications \& Services, pp. 177-186, 2012. DOI: 10.1145/2428736.2428765.

[88] R. Dube. "Process-oriented Complete Requirement Engineering Cycle for Generic Projects". Proceedings of the International Conference and Workshop on Emerging Trends in Technology, pp. 194-197, 2010. DOI: $10.1145 / 1741906.1741948$.

[89] V. Gervasi. "Reasoning About Inconsistencies in Natural Language Requirements". ACM Transactions on Software Engineering and Methodology (TOSEM). Vol. 14, pp. 277-330, 2007. DOI:10.1145/1072997.1072999.

[90] M. Giacalone, F. Massacci, F. Paci, and R. Perugino. "Security Triage: An Industrial Case Study on the Effectiveness of a Lean Methodology to Identify Security Requirements". Proceedings of the 8th ACM/ IEEE International Symposium on Empirical Software Engineering and Measurement, p. 24,2014 . DOI: $10.1145 / 2652524.2652585$. 
[91] N. Mitakides and N. Spanoudakis. "Validating Business Requirements Using MAS Analysis Models". Proceedings of the 2015 International Conference on Autonomous Agents and Multiagent Systems, pp. 1693-1694, 2015. DOI: ISBN: 978-1-4503-3413-6.

[92] A. Saini. "Weak Eyesight Therapy: A case study in designing an application for $\mathrm{m}$-Health systems". Human Computer Interactions (ICHCI), pp. 1-8. 2013. DOI: 10.1109/ ICHCI-IEEE.2013.6887804.

[93] F. Rodríguez and E. Grampin. "WekaTIE, a traffic classification plugin integrating TIE and Weka". Wireless Communications and Mobile Computing Conference (IWCMC), pp. 623-628. 2014. DOI: 10.1109/ IWCMC.2014.6906428. 